\title{
Antimicrobial Resistance in Enterobacterales Bacilli Isolated from Bloodstream Infection in Surgical Patients of Polish Hospitals
}

\author{
M. Kłos $\mathbb{D}^{1},{ }^{1}$ M. Pomorska-Wesołowska, ${ }^{2}$ D. Romaniszyn $\mathbb{D}^{3},{ }^{3}$ J. Wójkowska-Mach $\mathbb{D}^{3},^{3}$ \\ and A. Chmielarczyk $\mathbb{B}^{3}$ \\ ${ }^{1}$ Faculty of Health Sciences, Jagiellonian University Medical College, Kraków, Poland \\ ${ }^{2}$ Department of Microbiology, Analytical and Microbiological Laboratory, KORLAB, Ruda Slaska, Poland \\ ${ }^{3}$ Department of Microbiology, Faculty of Medicine, Jagiellonian University Medical College, Kraków, Poland
}

Correspondence should be addressed to M. Kłos; klosmartha@gmail.com

Received 5 October 2020; Revised 12 December 2020; Accepted 5 January 2021; Published 16 January 2021

Academic Editor: Carlo Genovese

Copyright ( $) 2021$ M. Kłos et al. This is an open access article distributed under the Creative Commons Attribution License, which permits unrestricted use, distribution, and reproduction in any medium, provided the original work is properly cited.

\begin{abstract}
Background and Aims. Bloodstream infections (BSIs) are one of the most frequently observed hospital-acquired infections (HAIs). We sought to describe the epidemiology and drug resistance secondary Enterobacterales BSIs in surgical patients and check for any correlation with the type of hospital ward. Materials and Methods. This multicenter (13 hospitals in southern Poland) laboratorybased retrospective study evaluated adults diagnosed with BSI secondary to surgical site infection (SSI) hospitalized in 2015-2018; 121 Enterobacterales strains were collected. The drug resistance was tested according to the EUCAST recommendations. Tests confirming the presence of extended-spectrum $\beta$-lactamases (ESBLs) and bla resistance genes were carried out. The occurrence of possible clonal epidemics among K. pneumoniae strains was examined. Results. The prevalence of Enterobacterales in secondary BSI was $12.1 \%$; the most common strains were E. coli $(n=74,61.2 \%)$ and Klebsiella spp. $(n=33,27.2 \%)$. High resistance involved ampicillin and ampicillin/sulbactam (92, 8-100\%), fluoroquinolones (48-73\%), and most cephalosporins (29-50\%). Carbapenems were the antimicrobials with the susceptibility at $98 \%$. The prevalence of ESBL strains was $37.2 \%(n=45)$. All the ESBL strains had $b l a_{\text {CтХ-м }}$ gene, $26.7 \%$ had the bla $a_{\text {SHV }}$ gene, and $24.4 \%$ had bla $a_{\text {TEM }}$ gene. The diversity of Klebsiella strains was relatively high. Only 4 strains belonged to one clone. Conclusions. What is particularly worrying is the high prevalence of Enterobacterales in BSI, as well as the high resistance to antimicrobial agents often used in the empirical therapy. To improve the effectiveness of empirical treatment in surgical departments, we need to know the epidemiology of both surgical site infection and BSI, secondary to SSI. We were surprised to note high heterogeneity among $K$. pneumoniae strains, which was different from our previous experience.
\end{abstract}

\section{Introduction}

In recent years, data concerning the epidemiology of bloodstream infections (BSI) have indicated a change in the prevalence of their microbial etiological agents. Gramnegative pathogens are predominant as the major causes of bacteremia, and according to researchers, the proportion of Escherichia coli increased by $33 \%$ and amounted to as many as $1 / 4$ of all pathogens and among patients of over 75 years of age, even by over 30\% [1]. Enterobacterales bloodstream infection (BSI) is one of the most frequently observed hospital-acquired infections (HAIs), especially in surgical patients. All over the world, also in Poland, there are significantly more secondary BSIs, a fact that calls for immediate measures taken in order to prevent and control BSI in adults.

In 1983, strains with the extended-spectrum $\beta$-lactamase (ESBL) mechanism were first observed, and this phenotype quickly became dominant among Gram-negative resistant strains. Genes transmitted mainly by plasmids are responsible for resistance to penicillins, cephalosporins, and aztreonam, and their presence is also associated with 
resistance to antibiotics other than $\beta$-lactams, i.e., fluoroquinolones or aminoglycosides [2]. For more than twenty years, an increase in the number of resistant strains and infections with ESBL strains has been observed [2]. Widespread use of inexpensive antibiotics such as cephalosporins leads to the spread of ESBL strains in Europe and the USA [3]. Among the ESBL strains, E. coli and Klebsiella pneumoniae most often cause BSI [2, 4].

Studies by Sakellariou et al. have shown that E.coli BSI is most often of urological origin, whereas K. pneumoniae BSI is associated with surgical site infection (SSI), respiratory tract infection, or unknown infection [5]. Factors increasing the risk of such infections include the presence of invasive devices, especially vascular or indwelling catheters, age, ICU stay, septic shock, stay in a nursing home, and previous antibiotic therapy or inappropriate therapy $[4,6]$. Antibiotic therapy with third-generation cephalosporins, $\beta$-lactams, and fluoroquinolones is particularly significant in the development of the ESBL phenotype [4]. ESBL infections are associated with increased mortality, costs, and prolonged hospitalization [2]. In the case of ESBL-positive $K$. pneumoniae, associated with surgical site infection, the risk factors include peritonitis, the use of broad-spectrum cephalosporins, neutropenia, septic shock, and ICU stay [7].

The main purpose of this study was to determine the drug resistance in Enterobacterales isolated from secondary bloodstream infection in surgical patients. In this manuscript, only cases of laboratory-confirmed bloodstream infections (LC-BSIs) were subjected to analysis according to the ECDC (European Centre for Disease Prevention and Control) definitions, in which it is diagnosed when the patient demonstrates at least one of the following symptoms: fever $\left(>38^{\circ} \mathrm{C}\right)$, chills, blood pressure drop, blood cultures giving at least one positive result and a pathogenic microorganism is isolated, or two independent trials, up to 48 hours apart, give rise [8]. Additionally, the occurrence of possible clonal epidemics among $K$. pneumoniae strains was examined.

\section{Materials and Methods}

This multicenter laboratory-based retrospective study included 997 adult surgery patients $(18 \leq)$ with LC-BSIs hospitalized in southern Poland (13 hospitals). The patients were hospitalized in 15 surgery units of different types: general, urology and vascular surgery wards, or gynecology and obstetrics wards. The qualifying criterion was to diagnose secondary bloodstream infection, except for central or peripheral venous catheter BSI.

Altogether, 121 Enterobacterales strains were isolated. Identification of microorganisms was performed using the MALDI-TOF Biotyper (Bruker Corporation, the Netherlands) according to standard methods. The isolates had been collected retrospectively in collaboration with KORLAB between 1 January 2015 and 31 December 2018 and the Department of Microbiology at the Jagiellonian University Medical College.

Antimicrobial susceptibility testing of all isolates was performed according to the current guidelines of the
European Committee on Antimicrobial Susceptibility Testing (EUCAST, http://www.eucast.org/ clinical_breakpoints/; accessed December 2017) by disc diffusion on Müeller-Hinton agar plates. Resistance was determined to six categories of antimicrobial agents: penicillin (also with clavulanic acid and tazobactam), cephalosporins (fourth-generation cefuroxime, ceftazidime, cefotaxime, cefepime, and cefoperazone-sulbactam), carbapenems (ertapenem, imipenem, and meropenem), aminoglycosides (amikacin, gentamicin, and tobramycin), fluoroquinolones (ciprofloxacin), and sulfamethoxazole with trimethoprim.

ESBL activity was detected with a modified double disk synergy test using a combination of cefotaxime, ceftazidime, cefepime, and aztreonam discs, placed $20 \mathrm{~mm}$ apart around a disc containing amoxicillin/clavulanic acid [9].

All isolates with ESBL activity were screened with multiplex PCR for the presence of $b l a_{\mathrm{TEM}}, b l a_{\mathrm{SHV}}$, and $b l a_{\text {OXA }} \beta$-lactamase genes and for variants of $b l a_{\mathrm{CTX}-\mathrm{M}}$ $\beta$-lactamase genes (group including $b l a_{\mathrm{CTX}-\mathrm{M}-1}$, bla $a_{\mathrm{CTX}-\mathrm{M}-3}$, and $b l a_{\mathrm{CTX}-\mathrm{M}-15}$, group 2 including $b l a_{\mathrm{CTX}-\mathrm{M}-2}$, and group 9 including $b l a_{\mathrm{CTX}-\mathrm{M}-9}$ and $\left.b l a_{\mathrm{CTX}-\mathrm{M}-14}\right)$ using previously published primers [10]. Due to the largest number of ESBLs among Klebsiella spp. being Klebsiella pneumoniae strains $(n=27)$, pulsed-field gel electrophoresis (PFGE) was performed to check for a clonal epidemic. Our previous experiments showed that Klebsiella strains isolated in Polish departments often belong to one clone. PFGE was used to determine the possible horizontal transfer of K. pneumoniae strains among patients from the same wards. Genomic DNA was digested with $10 \mathrm{U}$ XbaI (EURx, Gdańsk, Poland). The resulting DNA fingerprinting was obtained using the $\mathrm{CHEF}$ III PFGE system (BioRad, Warsaw, Poland) in 0.5 Trisborate-EDTA buffer at $14^{\circ} \mathrm{C}$ at $6 \mathrm{~V}$ for $22 \mathrm{~h}$ with a ramped pulse time of 2-35 s. The GelCompar (Applied Maths) was used for cluster analysis using the Dice coefficient and the unweighted pair group method with arithmetic mean.

\section{Results}

The median age (quartiles Q1 and Q3) was 67 years $(59,76)$, and $63(52.0 \%)$ patients were women. Most patients (47 persons, $38.8 \%$ ) were hospitalized in general surgery wards. In the range of less than 59 years, a higher number of cases among women were observed compared to men $(39.6 \%$ and $13.7 \%$, respectively); while with age, there is an increase in the number of cases among men (in the range of over 70 years: men $51.7 \%$, women $26.9 \%$ ).

The prevalence of Enterobacterales in secondary BSI was $12.1 \%$; the most common strain was $E$. coli $(n=74,61.2 \%)$ and Klebsiella spp. (33 patients, 27.2\%), regardless of the gender or age group (Table 1). In the genus Klebsiella, 27 Klebsiella pneumoniae, 5 Klebsiella oxytoca, and 1 Klebsiella mobilis strains were identified. An inconsiderable percentage of other species of Enterobacterales bacilli was $11.5 \%$. In the group designated “others," 10 strains were Proteus mirabilis, 3 Enterobacter cloacae, and 1 Citrobacter freundii.

The highest drug resistance was reported with regard to ampicillin in association with sulbactam, reaching 100\% 
TABLe 1: Prevalence of Enterobacterales bacilli in studied surgical wards amount 997 patients with BSI.

\begin{tabular}{|c|c|c|c|c|}
\hline \multirow{2}{*}{ Studies isolates } & \multicolumn{3}{|c|}{ Enterobacterales } & \multirow{2}{*}{ All $n=121$} \\
\hline & E. $\operatorname{coli} n=74$ & Klebsiella spp. $n=33$ & Others $n=14$ & \\
\hline Prevalence rate* $(\%)$ & 7.4 & 3.3 & 1.4 & 12.1 \\
\hline \multicolumn{5}{|l|}{ Surgery wards } \\
\hline General ward & $51(68.9 \%)$ & $27(81.8 \%)$ & $9(64.3 \%)$ & $87(71.9)$ \\
\hline Urology ward & $19(25.7 \%)$ & $5(15.1 \%)$ & $3(21.4 \%)$ & $27(22.3)$ \\
\hline Gynecology and obstetrics ward & $2(2.7 \%)$ & $1(3.0 \%)$ & $1(7.1 \%)$ & $4(3.3)$ \\
\hline Vascular ward & $2(2.7 \%)$ & $0(0.0 \%)$ & $1(7.1 \%)$ & $3(2.5)$ \\
\hline \multicolumn{5}{|l|}{ Age range } \\
\hline$\leq 59$ & $22(29.7 \%)$ & $6(18.1 \%)$ & $5(35.7 \%)$ & $33(27.3)$ \\
\hline $60-69$ & $22(30 \%)$ & $16(48 \%)$ & $3(21 \%)$ & $41(33.9)$ \\
\hline $70 \leq$ & $30(40.5 \%)$ & $11(33.3 \%)$ & $6(42.8 \%)$ & $47(38.8)$ \\
\hline Total & $74(61.1 \%)$ & $33(27.2 \%)$ & $14(11.5 \%)$ & $121(100)$ \\
\hline
\end{tabular}

*Calculated by dividing the number of strains by the number of laboratory-confirmed bloodstream infections and multiplying by 100 .

TABLE 2: Drug resistance in Enterobacterales bacilli in studied surgical wards.

\begin{tabular}{|c|c|c|c|c|c|}
\hline \multirow[b]{2}{*}{ Nr category } & \multirow[b]{2}{*}{ Antimicrobial category } & \multirow[b]{2}{*}{ Antimicrobial agent } & \multicolumn{3}{|c|}{ Enterobacterales $n=121(100 \%)$} \\
\hline & & & $\begin{array}{l}\text { E. coli } n=74 \\
(61.1 \%)\end{array}$ & $\begin{array}{c}\text { Klebsiella spp. } n=33 \\
(27.2 \%)\end{array}$ & $\begin{array}{c}\text { Others } n=14 \\
(11.5 \%)\end{array}$ \\
\hline \multirow{4}{*}{1} & \multirow{4}{*}{$\begin{array}{l}\text { Penicillins and penicillins with } \\
\text { inhibitors }\end{array}$} & \multirow{4}{*}{$\begin{array}{c}\text { Ampicillin } \\
\text { Ampicillin-sulbactam } \\
\text { Amoxicillin-clavulanic } \\
\text { acid } \\
\text { Piperacillin-tazobactam }\end{array}$} & $73(98.6 \%)$ & $33(100 \%)$ & $14(100 \%)$ \\
\hline & & & $72(97.2 \%)$ & $33(100 \%)$ & $13(92.8 \%)$ \\
\hline & & & $34(45.9 \%)$ & $20(60.6 \%)$ & $5(35.7 \%)$ \\
\hline & & & $10(13.5 \%)$ & $11(33.3 \%)$ & $3(21.4 \%)$ \\
\hline \multirow{5}{*}{2} & \multirow{5}{*}{ Cephalosporins } & Cefuroxime IV & $29(39.1 \%)$ & $15(45.4 \%)$ & $7(50.0 \%)$ \\
\hline & & Ceftazidime & $29(39.1 \%)$ & $14(42.4 \%)$ & $5(35.7 \%)$ \\
\hline & & Cefotaxime & $28(37.1 \%)$ & $13(39.3 \%)$ & $5(35.7 \%)$ \\
\hline & & Cefepime & $22(29.7 \%)$ & $16(48.4 \%)$ & $5(35.7 \%)$ \\
\hline & & Cefoperazone-sulbactam & $6(8.1 \%)$ & $6(18.1 \%)$ & 0 \\
\hline \multirow{3}{*}{3} & \multirow{3}{*}{ Carbapenems } & Ertapenem & 0 & $1(3.0 \%)$ & 0 \\
\hline & & Imipenem & $1(1.3 \%)$ & 0 & 0 \\
\hline & & Meropenem & 0 & 0 & 0 \\
\hline 4 & Fluoroquinolones & Ciprofloxacin & $36(48.6 \%)$ & $24(72.7 \%)$ & $8(57.1 \%)$ \\
\hline \multirow{3}{*}{5} & \multirow{3}{*}{ Aminoglycosides } & Gentamicin & $19(25.6 \%)$ & $7(21.2 \%)$ & $5(35.7 \%)$ \\
\hline & & Amikacin & $11(14.8 \%)$ & $13(39.3 \%)$ & $2(14.2 \%)$ \\
\hline & & Tobramycin & $24(32.4 \%)$ & $17(51.5 \%)$ & $5(35.7 \%)$ \\
\hline 6 & Others & $\begin{array}{l}\text { Trimethoprim- } \\
\text { sulfamethoxazole }\end{array}$ & $37(50.0 \%)$ & $21(63.6 \%)$ & $9(64.2 \%)$ \\
\hline \multirow{7}{*}{\multicolumn{2}{|c|}{ Nonsusceptible to antimicrobial categories* }} & $\begin{array}{l}0 \text { categories (fully } \\
\text { susceptible) }\end{array}$ & 0 & 0 & 0 \\
\hline & & 1 category & $25(33.7 \%)$ & $5(15.1 \%)$ & $2(14.2 \%)$ \\
\hline & & 2 categories & $15(20.2 \%)$ & $6(18.1 \%)$ & $3(21.4 \%)$ \\
\hline & & 3 categories & $4(5.4 \%)$ & $4(12.1 \%)$ & $4(28.5 \%)$ \\
\hline & & 4 categories & $11(14.8 \%)$ & $4(12.1 \%)$ & $1(7.1 \%)$ \\
\hline & & 5 categories & $18(24.3 \%)$ & $14(42.4 \%)$ & $4(28.5 \%)$ \\
\hline & & 6 categories & $1(1.36 \%)$ & 0 & 0 \\
\hline
\end{tabular}

*Strains were divided into six categories based on their resistance to a specific number of antimicrobial agent categories (penicillin and penicillins with inhibitors, cephalosporins, carbapenems, fluoroquinolones, aminoglycosides, and trimethoprim-sulfamethoxazole); fully susceptibility to all antimicrobial agents from all categories means "0"; " 6 " means resistance to at least one antimicrobial agent from each of the 6 categories.

(Table 2). High resistance also involved fluoroquinolones (48-73\%) and most cephalosporins (29-50\%). Only $1(0.8 \%)$ strain was fully sensitive, but 37 strains $(30.6 \%)$ were resistant to 5 or more antimicrobial categories studied (Table 2). The studied strains demonstrated almost full sensitivity only to carbapenems (nearly $100 \%$ sensitivity), and two strains (E. coli and Klebsiella pneumoniae) were observed to be resistant to selected carbapenems.

The prevalence of ESBL strains was $37.2 \%(n=45)$. Most ESBL-positive strains belonged to the genus Klebsiella $(n=22 ; 66.7 \%)$ and were especially present in general surgery wards $(n=175 ; 1.5 \%)$. Among $E$. coli, although they 
TABle 3: Prevalence of ESBL strains among Enterobacterales bacilli in studied surgical wards.

\begin{tabular}{|c|c|c|c|c|}
\hline Studied isolates & E. $\operatorname{coli} n=74(100 \%)$ & Klebsiella spp. $n=33(100 \%)$ & Others $n=14(100 \%)$ & All isolates $n=121(100 \%)$ \\
\hline ESBL (yes, \%) & $19(25.7 \%)$ & $22(66.7 \%)$ & $4(28.6 \%)$ & $45(37.2 \%)$ \\
\hline General ward & $15(20.3 \%)$ & $17(51.5 \%)$ & $3(21.4 \%)$ & $35(28.9 \%)$ \\
\hline Urology ward & $3(4.0 \%)$ & $4(12.1 \%)$ & $1(7.1 \%)$ & $8(6.6 \%)$ \\
\hline Gynecology and obstetrics ward & $1(1.4 \%)$ & $1(3.0 \%)$ & $0(0 \%)$ & $2(1.6 \%)$ \\
\hline Vascular ward & $0(0 \%)$ & $0(0 \%)$ & $0(0 \%)$ & $0(0 \%)$ \\
\hline
\end{tabular}

constitute the majority of the Enterobacterales strains isolated, ESBL strains were about $1 / 4$ (Table 3).

Among the ESBL $(n=45)$ strains, 12 strains $(26.7 \%)$ had the bla $a_{\mathrm{SHV}}$ gene (10 Klebsiella and 2 others), 11 strains $(24.4 \%)$ had bla $a_{\text {TEM }}(6$ Klebsiella, 3 others, and 2 E. coli), 14 strains $(31.1 \%)$ had bla OXA $(6$ E. coli, 6 Klebsiella, and 2 others), and 45 strains (100\%) had bla $a_{\text {CTX-M }}$ (Table 3).

The most common were bla $a_{\mathrm{CTX}-\mathrm{M}}$ genes belonging to group 1 (including $b l a_{\mathrm{CTX}-\mathrm{M}-1}, b l a_{\mathrm{CTX}-\mathrm{M}-3}$, and $b l a_{\mathrm{CTX}-\mathrm{M}-15}$ ) up to 34 strains $(75.5 \%)$, then variants of $b l a_{\mathrm{CTX}-\mathrm{M}}$ group 9 (including $b l a_{\mathrm{CTX}-\mathrm{M}-9}$ and $\left.b l a_{\mathrm{CTX}-\mathrm{M}-14}\right)$ in 4 strains $(8.9 \%)$, and group 2 (including $b l a_{\mathrm{CTX}-\mathrm{M}-2}$ ) in one strain $(2.2 \%)$. Seven of the $b l a_{\text {CTX-M }}$ genes belonged to groups other than those tested.

The result of PFGE, among the studied Klebsiella pneumoniae strains, showed that only 4 strains that have been derived from the same ward of general surgery belonged to one clone. Other strains varied widely (Figure 1).

\section{Discussion}

Enterobacterales infections are common in surgical wards, but the presented data show that their prevalence depends significantly on the type of surgery procedures. In the analyzed population, they were most often identified in general surgery and after urological procedures, while in other populations, this type of etiology was rare.

According to Kolpa et al., the prevalence of Enterobacteriaceae in HAIs in patients after neurosurgical procedures was $25.8 \%$, but mainly related to BSI cases, where prevalence was as high as $29.8 \%$ [11]. Similar values (33\%) were obtained in pediatric cardiac surgery [12]. And as expected, completely different results were obtained in studies on infections in patients after urological procedures, where Enterobacteriaceae in BSI constituted 67.9\%. The strains from LC-BSI showed significant drug resistance. They were highly susceptible only to ciprofloxacin, piperacillin-tazobactam (90\%), meropenem, and gentamicin (100\%) [13].

According to Melzer et al., urinary catheters increase the risk of severe bacteremia and should be used only when there is a clinical indication [14]. Research results by Lillie et al. confirm that one of the main reasons for the increase in BSI is the use of urinary catheters, often unnecessary [15]. Probably, the best way to reduce the number of BSI among urological patients is to reduce the indwelling catheters utilization and shortening the urinary catheter use [16].

To improve the effectiveness of empirical treatment in surgical departments, we need to know the epidemiology of both surgical site infection and BSI secondary to SSI, including the prevalence of their microbial etiological agents and antibiotic resistance profiles. Our results indicate an alarmingly high level of drug resistance in Enterobacterales strains associated with secondary BSIs in surgery patients in southern Poland. This situation may affect the risk of therapeutic failures in empirical therapy. Based on presented data in studied population, effective empirical therapy is possible only with cefoperazone-sulbactam or piperacillintazobactam in combination with aminoglycosides or the use of carbapenems, which is definitely unfavorable because it carries a high risk of increasing resistance to carbapenems among Enterobacterales which is already a big problem in other European countries such as Romania, Greece, and Italy [17]. In Poland, among E. coli and K. pneumoniae strains, resistance to aminopenicillins, third-generation cephalosporins, and fluoroquinolones is still a bigger problem, which does not mean that we should not be afraid of the appearance of carbapenem-resistant strains [18]. Fortunately, resistance to carbapenems in Enterobacterales was not confirmed in our studies, as only one strain was resistant to imipenem. However, the application of one of the antibiotics from the group of $\beta$-lactams, carbapenems, aminoglycosides, or fluoroquinolones may lead to coselection resistance to several groups of antibiotics. This is an indication for the need to perform microbiological tests concurrently with targeted therapy and to conduct precise microbiological surveillance. Unfortunately, in Polish hospitals, microbiological testing is underutilized and antibiotic consumption is one of the highest in Europe [19]. On the other hand, measures are required to implement appropriate infection prevention and control procedures.

Despite the fact that no mechanisms of carbapenem resistance have been found in our study, we still observe a high level of ESBL-positive strains, especially in $K$. pneumoniae. This observation is consistent with reports from the SENTRY Antimicrobial Surveillance Program, which indicate a continuous and faster increase in the share of ESBL-positive K. pneumoniae and E. coli strains in various types of infections in hospitals in the US [20]. In our 2011-2013 research, the presence of ESBL was only slightly lower at $57 \% \mathrm{KP}$ and $21.4 \% \mathrm{EC} \mathrm{[21].} \mathrm{In}$ addition, ESBL strains isolated from newborns were resistant to aminoglycosides and SXT as here. Molecular characterization of the 45 ESBL-producing isolates showed that all $(100 \%)$ isolates were producing $b l a_{\mathrm{CTX}-\mathrm{M}^{-}}$ type ESBLs, which represented most of all by groups $b l a_{\mathrm{CTX}-\mathrm{M}-1}, b l a_{\mathrm{CTX}-\mathrm{M}-3}$, and $b l a_{\mathrm{CTX}-\mathrm{M}-15}(75.5 \%)$, which is consistent with our other studies about ESBL strains in southern Poland [21, 22]. 


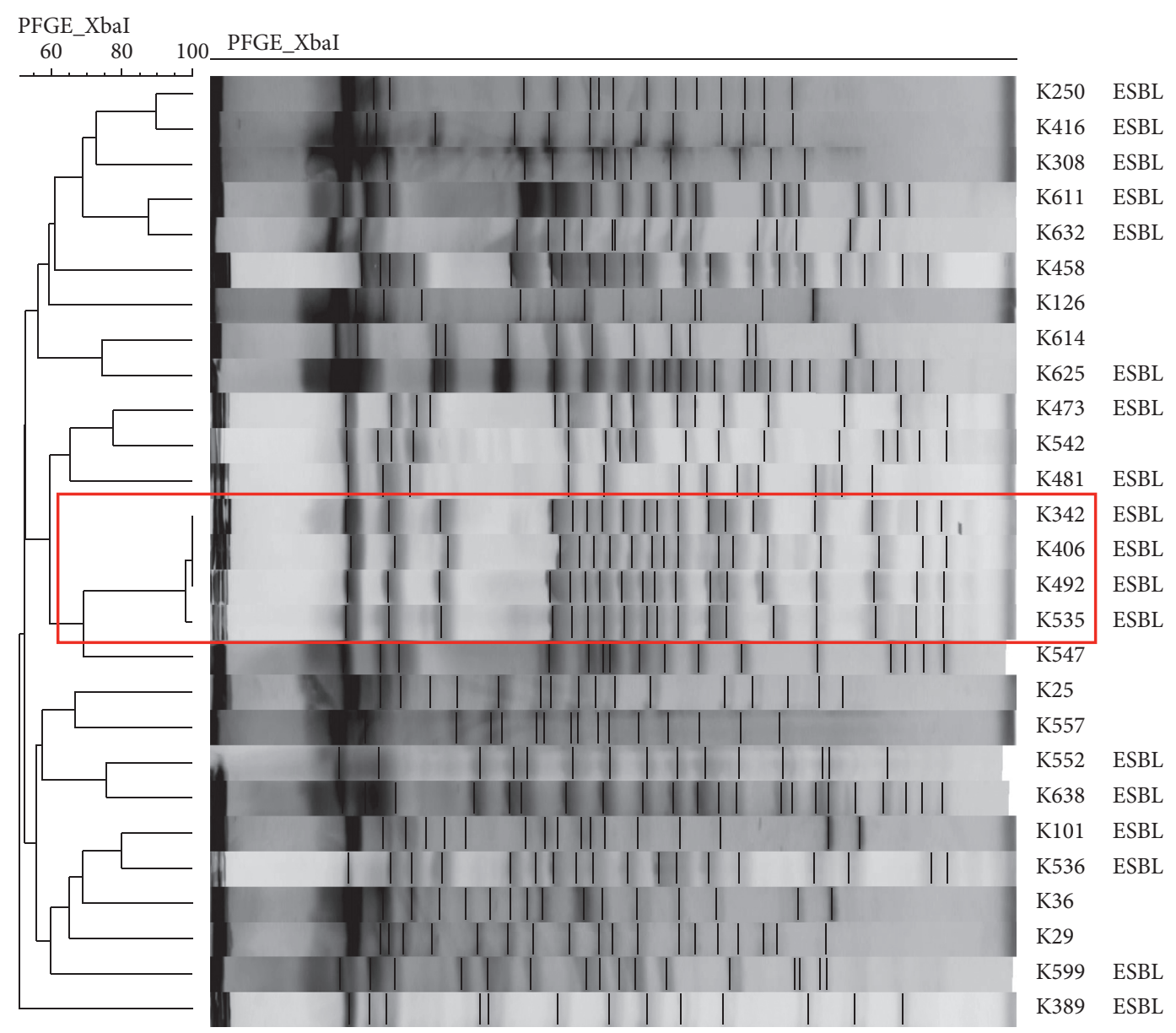

FIgURe 1: Pulsed-field gel electrophoresis dendrogram of Klebsiella pneumoniae strains.

Molecular studies suggest that both mechanisms (clonal spread and plasmid transfer) are important since ESBLpositive strains have a larger share of the outbreak than others. The abundance of ESBL-positive strains from materials (wound, blood) from surgical patients may also be due to the aggressive broad-spectrum antibiotic policy. We should not ignore the local prevalence of ESBL when drug delivery algorithms are constructed.

Based on previous experience concerning research in Polish hospitals, we know that clonal spread more often affects Klebsiella pneumoniae strains than E. coli; hence, we have now checked whether the high ESBL share among this species is not associated with clonal outbreak $[21,23]$. However, the diversity of Klebsiella strains in PFGE was relatively high-only 4 strains belonged to one clone. This fact was different from our previous experience; probably, the cases of infection in this study were not associated with the horizontal transfer.

The high prevalence of ESBLs among Enterobacterales in hospital wards cannot be unambiguously associated with a high percentage of ESBLs in the general population, and studies conducted by $\mathrm{Ny}$ et al. among volunteers in six European countries, including Poland, showed a prevalence of $8 \%$ in the case of E. coli ESBL and $0 \%$ in $K$. pneumoniae ESBL among polish carriers in Silesian Voivodeship [24].

In the light of the data presented, rapid implementation of targeted treatment, especially based on sensitive and quick diagnostic tests, is of particular importance for successful infection treatment. In addition to species identification, more and more commercially available kits for identifying bacteria from blood by molecular methods are able to detect the most popular resistance mechanisms, e.g., KPC, NDM, bla $a_{\mathrm{OXA}-48}$, and others. In Poland, there are currently no clear recommendations on how to effectively implement this type of procedure, and for many hospitals and laboratories, the costs of modern tests may also be a barrier [25]. Our study has a few limitations. It is generally assumed that $E$. coli and K. pneumoniae BSI behave similarly, since such a combination was commonly adopted in the literature. Clinical data regarding the hospitalization period were available, and we could analyze 
only the in-hospital outcome. Previous history of hospitalization was unknown, and we did not know if the patient had previously used antibiotics or had an underlying disease.

\section{Data Availability}

The data used to support the findings of this study are available from the Department of Microbiology of the Jagiellonian University Medical College upon request (Agnieszka Chmielarczyk, agnieszka.chmielarczyk@uj.edu.pl and Jadwiga Wojkowska-Mach, jadwiga.wojkowskamach@uj.edu.pl). Data on drug resistance of strains are also available in the KORLAB laboratory (Monika PomorskaWesołowska monikapw@op.pl).

\section{Conflicts of Interest}

The authors declare that they have no conflicts of interest.

\section{Authors' Contributions}

ACh and JWM have conceptualised and designed the study; MP-W collected the data; DR and MK performed the phenotypic and genotypic analyses; ACh and JWM analyzed and interpreted the data; MK, ACh, and JWM wrote the manuscript; Ach and JWM reviewed and revised it and gave the final approval of the version to be published.

\section{Acknowledgments}

This work was supported by a grant from the Jagiellonian University Medical School (N41/DBS/000053).

\section{References}

[1] J. Wilson, S. Elgohari, D. M. Livermore et al., "Trends among pathogens reported as causing bacteraemia in England, 20042008," Clinical Microbiology and Infection, vol. 17, no. 3, pp. 451-458, 2011.

[2] M. J. Schwaber, S. Navon-Venezia, K. S. Kaye, R. Ben-Ami, D. Schwartz, and Y. Carmeli, "Clinical and economic impact of bacteremia with extended-spectrum- $\beta$-lactamase-producing Enterobacteriaceae," Antimicrobial Agents and Chemotherapy, vol. 50, no. 4, pp. 1257-1262, 2006.

[3] Y. Ikeda, T. Mamiya, H. Nishiyama et al., "Risk factors for extended-spectrum $\beta$-lactamase-producing Escherichia coli infection in hospitalized patients," Nagoya Journal of Medical Science, vol. 74, no. 1-2, pp. 105-114, 2012.

[4] T. Chopra, D. Marchaim, P. C. Johnson et al., "Risk factors for bloodstream infection caused by extended-spectrum $\beta$-lactamase-producing Escherichia coli and Klebsiella pneumoniae: a focus on antimicrobials including cefepime," American Journal of Infection Control, vol. 43, no. 7, pp. 719-723, 2015.

[5] C. Sakellariou, S. Gürntke, I. Steinmetz et al., "Sepsis caused by extended-spectrum beta-lactamase (ESBL)positive K. pneumoniae and E. coli: comparison of severity of sepsis, delay of anti-Infective therapy and ESBL genotype," PLoS One, vol. 11, no. 7, 2016.

[6] O. Scheuerman, V. Schechner, Y. Carmeli et al., "Comparison of predictors and mortality between bloodstream infections caused by ESBL-producing Escherichia coli and ESBL- producing Klebsiella pneumoniae," Infection Control \& Hospital Epidemiology, vol. 39, no. 6, pp. 660-667, 2018.

[7] C.-I. Kang, S.-H. Kim, W. B. Park et al., "Bloodstream infections due to extended-spectrum $\beta$-lactamase-producing Escherichia coli and Klebsiella pneumoniae: risk factors for mortality and treatment outcome, with special emphasis on antimicrobial therapy," Antimicrobial Agents and Chemotherapy, vol. 48, no. 12, pp. 4574-4581, 2004.

[8] https://ecdc.europa.eu/sites/portal/files/documents/HAINet-ICU-protocol-v2.2_0.pdf.

[9] L. Drieux, F. Brossier, W. Sougakoff et al., "Phenotypic detection of extended-spectrum $\beta$-lactamase production in enterobacteriaceae: review and bench guide," Clinical Microbiology and Infection, vol. 14, no. 90-103, 2008.

[10] C. Dallene, A. Da Costa, D. Decré et al., "Development of a set of multiplex PCR assays for the detection of genes encoding important beta-lactamases in enterobacteriaceae," Journal of Antimicrobial Chemotherapy, vol. 65, no. 3, pp. 490-495, 2010.

[11] M. Kolpa, M. Wałaszek, A. Różańska et al., "Epidemiology of surgical site infections and non-surgical infections in neurosurgical polish patients-substantial changes in 2003-2017," International Journal of Environmental Research and Public Health, vol. 16, no. 6, p. 911, 2019.

[12] R. Abou Elella, H. K. Najm, H. Balkhy, L. Bullard, and M. S. Kabbani, "Impact of bloodstream infection on the outcome of children undergoing cardiac surgery," Pediatric Cardiology, vol. 31, no. 4, pp. 483-489, 2010.

[13] E. Kolwijck, A. E. M. Seegers, S. C. M. Tops et al., "Incidence and microbiology of post-operative infections after radical cystectomy and ureteral stent removal; a retrospective cohort study," BMC Infectious Diseases, vol. 19, no. 1, p. 303, 2019.

[14] M. Melzer and C. Welch, "Does the presence of a urinary catheter predict severe sepsis in a bacteraemic cohort?" Journal of Hospital Infection, vol. 95, no. 4, pp. 376-382, 2017.

[15] P. J. Lillie, G. Johnson, M. Ivan, G. D. Barlow, and P. J. Moss, "Escherichia coli bloodstream infection outcomes and preventability: a six-month prospective observational study," Journal of Hospital Infection, vol. 103, no. 2, pp. 128-133, 2019.

[16] T. M. Hooton, S. F. Bradley, D. D. Cardenas et al., "Diagnosis, prevention, and treatment of catheter-associated urinary tract infection in adults: 2009 international clinical practice guidelines from the infectious diseases society of America," Clinical Infectious Diseases, vol. 50, no. 5, pp. 625-663, 2010.

[17] https://atlas.ecdc.europa.eu/public/index.aspx? Dataset $=27 \&$ Health Topic $=4$.

[18] V. Jarlier, H. L. Diaz, O. E. Heuer et al., "Strong correlation between the rates of intrinsically antibiotic-resistant species and the rates of acquired resistance in gram-negative species causing bacteraemia, EU/EEA," Euro Surveillance, vol. 24, no. 33, 2019.

[19] J. Wojkowska-Mach, B. Godman, A. Glassman et al., “Antibiotic consumption and antimicrobial resistance in Poland; findings and implications," Antimicrobial Resistance and Infection Control, vol. 7, p. 136, 2018.

[20] M. Castanheira, J. C. Mills, S. E. Costello, R. N. Jones, and H. S. Sader, "Ceftazidime-avibactam activity tested against Enterobacteriaceae isolates from U.S. Hospitals (2011 to 2013) and characterization of $\beta$-lactamase-producing strains," $A n$ timicrobial Agents and Chemotherapy, vol. 59, no. 6, pp. 3509-3517, 2015.

[21] J. Wójkowska-Mach, A. Chmielarczyk, M. Borszewska-Kornacka et al., "Enterobacteriaceae infections of very low birth weight infants in polish neonatal intensive care units," The 
Pediatric Infectious Disease Journal, vol. 32, no. 6, pp. 594598, 2013.

[22] A. Chmielarczyk, M. Pobiega, J. Wójkowska-Mach et al., "Molecular epidemiology, plasmid analysis, virulence, and resistance of Escherichia coli isolated from neonatal intensive care units in Poland," Diagnostic Microbiology and Infectious Disease, vol. 76, no. 4, pp. 542-545, 2013.

[23] A. Chmielarczyk, M. Pobiega, J. Wójkowska-Mach, D. Romaniszyn, P. B. Heczko, and M. Bulanda, "Bloodstream infections due to Enterobacteriaceae among neonates in Poland - molecular analysis of the isolates," Polish Journal of Microbiology, vol. 64, no. 3, pp. 217-225, 2015.

[24] S. Ny, R. Kozlov, U. Dumpis et al., "Large variation in ESBLproducing Escherichia coli carriers in six European countries including Russia," European Journal of Clinical Microbiology \& Infectious Diseases, vol. 37, no. 12, pp. 2347-2354, 2018.

[25] D. R. Giacobbe, T. Giani, M. Bassetti et al., "Rapid microbiological tests for bloodstream infections due to multidrug resistant Gram-negative bacteria: therapeutic implications," Clinical Microbiology and Infection, vol. 26, no. 6, pp. 713-722, 2020. 